\title{
The Effects of Emotional Abuse and Neglect in Adulthood
}

\author{
Alyssa Jimenez $^{1 *}$
}

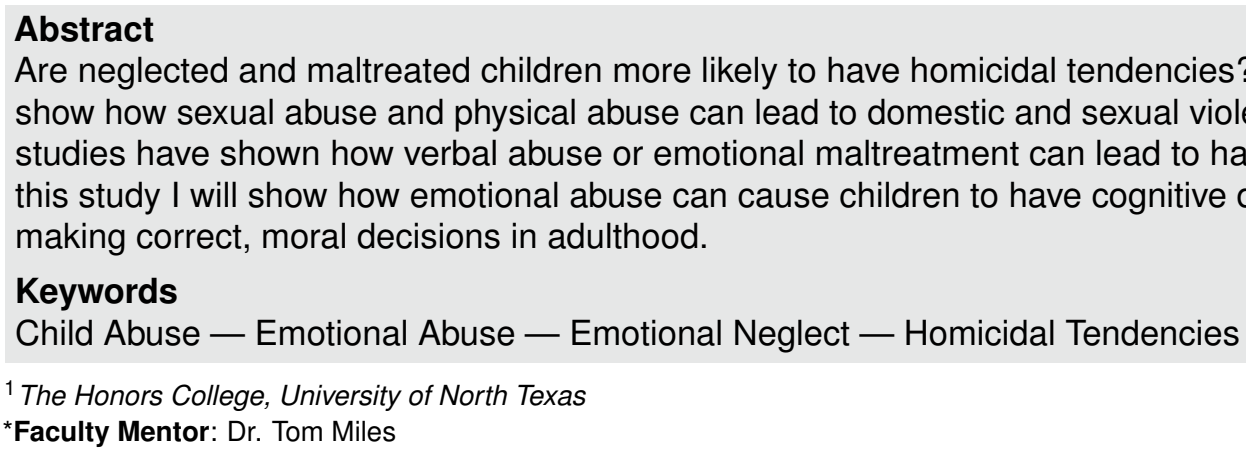

\section{Contents}

Introduction

1 The Cycle of Violence

2 Childhood Predictors

3 Results

4 Conclusion

References

\section{Introduction}

Over the last few years, sexual abuse and physical abuse have been at the forefront of the discourse on child development, but many seem to forget about emotional abuse and neglect. Emotional abuse, neglect, and child maltreatment are all problems that can cause one to develop cognitive issues and eventually can lead to violent crimes being committed in their adulthood. Emotional abuse can be anything from intimidation, blaming of the victim, isolating the victim, yelling, swearing, threats, name calling, and denial of abuse. These examples of emotional abuse, if done throughout a child's life, can cause harm.

Although not everyone sees yelling or swearing as forms of emotional abuse, they can cause psychological damage on the way children think. If these children are growing up in homes where they are living in constant fear and feeling as if they are worthless, then that will translate into their adulthood. Now, I am not predicting that every child who has been emotionally abused throughout their childhood will have any cognitive deficits or even be affected by the abuse, but there are cases in which this does happen. In the study, I will search to figure out if there is a connection between childhood

emotional abuse and neglect and homicidal tendencies and violent offenses in adulthood.

1

2

3

4

4

(1)

$$
\text { in }
$$
influence of childhood abuse history on adulthood executive functioning in offenders." Not only were they trying to understand this influence on executive functioning but also trying to see "whether executive performance is affected differentially by different abusive events." They were able to conclude that offenders who had a history of abuse, whether it was emotional, physical, or sexual, all seem to show poor cognitive flexibilities. This is relevant to the current study because "the emotionally abused participants have decits in executive control and set-switching abilities." Since these participants showed lack of executive controls this could possibly create an invitation for homicidal tendencies and ideations to creep into the minds of individuals who are emotionally abused or maltreated during their childhood.

In a similar study, researchers sought to figure out if children were maltreated, neglected, or emotionally abused during their childhood then would they be more crime prone later in life. Within the study they found that "the impulsive trait of urgency may play a significant role in linking emotional abuse to crime in young adulthood."'[9] By finding this connection they could understand that children with emotional abuse in their history commit a crime due to certain distresses. These distresses could be psychological but also that "childhood neglect is related to neurological deficits. Several studies have 
indicated that child neglect is related to altered brain development in regions that are involved in cognitive functioning." This altering brain development also connects with BecerraGarcia's study in a way that shows which crimes will be committed because the victims of emotional child abuse grow up not being able to sort out various aspects, or situations, in their brain. By not being able to sort out what exactly is right and wrong actions, then a child could potentially not know a certain behavior is wrong because they grew up thinking it was okay to do. An example of this could be a child that has a father who screams in their face every single day over the smallest issues. This child is constantly living in fear that they are going to be screamed at or slapped across the face will in turn become that fear-stricken child to grow up and do just those same actions to their children. Thus, leading to a never-ending cycle of abuse and violence.

Traumatizing childhood experiences can be related to psychopathy in offenders. Traumatizing experiences can include actions such as striking fear into the child, intimidation, and threats. Not limited to traumatizing childhood experiences but also "childhood victimization and maltreatment may increase a person's risk for psychopathy, which in turn, may put her or him at an increased risk for violent behavior.'[10] Childhood maltreatment consequences can affect personality which then can lead to them being more likely to develop violent behaviors. The neglect and maltreatment that some children receive during their childhood can lead to personality disorders which include psychopathy; which in turn could advance to impulsive and irresponsible behavior. This also "indicated that childhood victimization and maltreatment increased the risk of psychopathy in both female and male homicide oenders." This study has shown that there is a relationship between childhood maltreatment and violence which then increases the person to have developed psychopathic traits. Once these traits are developed they are more likely to be engaged in homicidal and violent behavior. This has indicated that childhood maltreatment, in the sense of verbal abuse and neglect, can have an effect in their adulthood which can make them more likely to offend. By having this cycle of violence, we are not creating a generation of new, positive relationships, but instead continuing the cycle.

\section{Childhood Predictors}

Physical abuse and maltreatment is a topic that has been studied tremendously by many researchers. However, this study focused more on the aspect of physical violence and harsh punishment regarding the likelihood that emotional abuse and childhood neglect will affect that child during adulthood. It was shown that physical punishment is associated with, and can potentially lead to, emotional abuse and emotional neglect which then can lead to someone experiencing intimate partner violence. This also showed that "physical punishment is related to other types of child maltreatment and violence across the lifespan.'[1] By having this information is it can be shown what predictors could there are in abuse cases and how this can affect adulthood. The results of this study were that "harsh physical punishment was associated with increased odds of childhood maltreatment, including emotional abuse, sexual abuse, physical abuse, physical neglect, emotional neglect." Thus, showing that if a teacher sees a child getting pushed by their parent, or a parent realizes that they have crossed the line, then this could be a predictor that emotional abuse and neglect could occur, if it is not already present. Having predictors like those shown in this study, show that there are signs before bad moments happen.

Additionally, in a separate study done by Ahonen, Loeber, Pardini in 2016, they too looked at some predictors of the childhood in violent offenders and non-violent individuals.[2] With this study though, they realized "that out of the 28 predictors tested in late childhood, 23 risk factors significantly discriminated between all violent offenders and non-violent individuals." Some of these risk factors included aspects such as physical abuse, physical punishment, troubled relationship between parent and child, and the "boy's counter control when disciplined." These predictors are not set in stone and are not assumed to be the only predictors that could lead to violent offenses. These are just some predictors that could cause emotional trauma, psychopathy, or mental issues which could lead to one being more likely to have violent thoughts or commit violent actions. Another connection between these two studies were "the strongest individual factors were covert behavior .... psychopathy/callous-unemotional traits;" thus, the psychopathy. By showing these unemotional traits, then that explains why someone children grow up to not care what decision they make because they lack tat empathy and emotion.

With all this information in mind, there are other ways that children can be emotionally abused. It can be "experiences such as teasing, rejecting, humiliating, and demeaning may contribute to the development of depressive cognitions and symptoms in children, regardless of whether these messages come from parents or peers.'[6] Experiencing these depressive cognitions can influence a child in ways that could make adult choice harder and makes them more likely to not be able to decipher between certain aspects. By going through this emotional abuse, or trauma, at an early age can have its consequences later down the road. In this study it was also shown "that emotional abuse and verbal victimization can contribute to the development of negative inferential styles." Similarly, to Gibb and Abela's study, Turner, Taillieu, Cheung, and Afifi performed a study in 2017, that showed "child maltreatment is associated with increased odds of mental disorders among males."[8] Developing a mental disorder can certainly be caused by environmental factors; I am not saying that environmental factors are the only cause, but they do play a role in mental disorders. In conclusion to this study, "emotional abuse, physical abuse, and exposure to IPV were the most common forms of maltreatment that co-occurred with CSA among males." They could present that childhood sexual abuse, along with other types of abuse, such as emotional abuse, makes those children more likely to develop a men- 
tal disorder than kids who weren't maltreated. Developing a mental disorder, and not having your brain acting like it should, can cause people to think odd thoughts. Those odd thoughts could potentially lead to not necessarily knowing what is exactly right and wrong.

Witnessing domestic violence can have some of the same effects as experiencing the direct violence. It was shown in a current study that "the relationship between witnessing domestic violence during childhood (i.e., witnessing, hearing, or intervening in abuse against a parent/sibling) and psychopathic traits in adulthood in a sample of 127 incarcerated male offenders."'[4] These traits can evidently lead to more crimes being committed as an adult and makes them more liking to offend and reoffend in adulthood. During childhood maltreatment, which includes physical/emotional abuse and neglect, there are some risk factors that can develop psychopathic traits. These adult offenders who experienced the maltreatment in during the childhood were more likely to gain these psychopathic traits, thus leading them to become adult offenders. The results of this study included that "traumatic events during childhood with psychopathic traits later in life, and suggest that domestic violence exposure may be one factor contributing to the manipulative, interpersonal style exhibited by individuals high in psychopathy." Psychopathy, a mental disorder, can play a role in homicidal tendencies or homicidal ideation. Although there is not a clear line that connects the two, there is a connection as to how some people have these thoughts and ideas in their mind while others do not.

Lastly, an influential study done in finding "that abused and neglected children have significantly greater risk of becoming delinquents, criminals, and violent criminals.'[11] The author showed she was showing us that there is a link between childhood victimization and later violent behavior in adult life, but it is not one hundred percent certain. In her finding she found "that it is important to distinguish factors that may stimulate an individual to become involved in crime from the factors that affect whether the person continues or desists in a criminal career." These studies are all showing that looking for predictors and being more aware that predictors do occur will be more beneficial to children during their childhood. Once the signs are starting to show early on, then the parents can start getting involved and do what is necessary to put the child on a better path.

Theory Children who are emotionally abused, neglected, and maltreated during childhood are more likely to have homicidal tendencies overall and have a higher possibility to develop cognitive deficits that prevent them from making correct, moral decisions in adulthood.

For this study, we used data from multiple resources including a Master Clinicals Review done by Journal of the American Academy of Child \& Adolescent Psychiatry and journal on The Long-Term Impact of the Physical, Emotional, and Sexual Abuse of Children: A Community Study. The first journal goes into physical, sexual, and emotional abuse on a more psychological and clinical level. This journal studied "more than 1,000 children 5 to 9 years old who were recruited from pediatric practices determined that those children who had been maltreated were nearly three times more likely to be diagnosed with an internalizing or externalizing disorder.'[12]

The second journal mentioned sent a postal questionnaire in the mail to 2,250 women and used a random sampling program to send them out.[7] On the postal questionnaire, there were questions that included the topics of general health, mental health, demographics, social support, and experience of sexual and physical abuse both as a child and during adult life. The next stage of this was the interview phase; this is when 298 women under the age of 65 that had marked that had been experienced unwanted sexual advances prior to the age of 16 were asked to come in for the interview. They measured the abuse in three different ways in this study. The first was exploring the childhood sexual abuse that each one went through and finding out, in much further detail, if there was a sexual touching or abuse involving actual penetration.

\section{Results}

When looking at the results of The Long-Term Impact of the Physical, Emotional, and Sexual Abuse of Children: A Community Study they found defined emotional abuse as "a failure of adequate care and concern rather than the active derogation or belittling of the child."[12] They were able to come to the result that" those women who reported having been exposed to some form of abuse in childhood, be it sexual, physical, or emotional were in adult life more likely to have mental health, interpersonal, and sexual difficulties." By having been exposed to one or all of these different types of abuse then it resulted in those women being more likely to have mental health difficulties which can overall lead to making poor choices that by-product could be having homicidal tendencies.

When looking at the results of the second journal article, a Master Clinicals Review done by Journal of the American Academy of Child \& Adolescent Psychiatry, they came to the conclusion that "child maltreatment-physical, sexual, and emotional abuse, as well as various forms of neglect-leads to increased risk for psychopathology across development, including internalizing disorders, externalizing disorders, trauma- and stress-related disorders, neurodevelopmental disorders (e.g. attention-deficit/hyperactivity disorder), and substance use disorders, as well as academic problems, delinquency, and difficulties in social relationships."[12] The journal found similar results to the first journal, in that the way a child is treated in their childhood does affect each individual in the long run. When maltreatment or emotional abuse occur, there leaves room for many different types of difficulties in adulthood including the for psychopathic development. I predict that there may not be a direct correlation between emotional abuse and homicidal tendencies, but I do believe there with be a connection between emotional abuse and violence and homicidal tendencies. 


\section{Conclusion}

In conclusion, I have found that emotional abuse can cause psychological deficits. Childhood maltreatment and emotional abuse can have an impact on a child's life in adulthood if they experience the emotional abuse. and These deficits can lead to children lacking emotion and not truly understanding the choices that individual is making. Thus, by not truly caring or understanding their choices, they are more likely to become a part of violent and criminal activities.

\section{References}

[1] Afifi, T. O., Mota, N., Sareen, J., \& MacMillan, H. L. (2017). The Relationships Between Harsh Physical Punishment and Child Maltreatment in Childhood and Intimate Partner Violence in Adulthood Public Health, 17, 1-10. doi:10.1186/s12889-017-4359-8

[2] Ahonen, L., Loeber, R., \& Pardini, D. (2016). The Prediction of Young Homicide and Violent Offenders. JQ: Justice Quarterly, 33(7), 1265-1291. doi:10.1080/ 07418825.2015 .1081263

[3] Becerra-García, J. A. (2014). Childhood Abuse and Neurocognitive Performance in Adulthood: A Preliminary Study of Executive Functioning in Offenders. Journal of Forensic Psychiatry \& Psychology, 25(1), 113-119. doi:10.1080/14789949.2013.873070

[4] Dargis, M., Koenigs, M. (2017). Witnessing Domestic Violence During Childhood is Associated with Psychopathic Traits in Adult Male Criminal Offenders. Law \& Human Behavior (American Psychological Association), 41(2), 173-179. doi:10.1037/1 hb0000226

[5] DuPre, D. P., \& Sites, J. (2015; 2015). Child Abuse Investigation Field Guide. Oxford; 4: Elsevier. Retrieved from http: //www. sciencedirect.com/ science/book/9780128023273.

[6] Gibb, B. E., \& Abela, J. R. Z. (2008). Emotional Abuse, Verbal Victimization, and the Development of Children's Negative Inferential Styles and Depressive Symptoms. Cognitive Therapy \& Research,32(2), 161-176. do i : 10 . $1007 / \mathrm{s} 10608-006-9106-x$.

[7] Mullen, P. E., et al. (1996). The Long Term Impact of the Physical, Emotional, and Sexual Abuse of Children: A Community Study. Pergamon, 20(1), 7-21.

[8] Turner, S., Taillieu, T., Cheung, K., \& Afifi, T. O. (2017). The Relationship Between Childhood Sexual Abuse and Mental Health Outcomes Among Males: Results From a Nationally Representative United States Sample. Child Abuse \& Neglect, 66, 64-72. doi: 10 . 1016/j.chiabu.2017.01.018.

[9] Shin, S. H., Cook, A. K., Morris, N. A., McDougle, R., \& Groves, L. P. (2016). The Different Faces of Impulsivity as Links Between Childhood Maltreatment and Young
Adult Crime. Preventive Medicine, 88, 210-217. doi : $10.1016 / j$.ypmed.2016.03.022.

[10] Weizmann-Henelius, G., Gronroos, M., Putkonen, H., Eronen, M., Lindberg, N., \& Hakkanen Nyholm, H. (2010). Psychopathy and gender differences in childhood psychosocial characteristics in homicide offenders - a nationwide register-based study. Journal of Forensic Psychiatry \& Psychology, 21(6), 801-814. doi: $10.1080 / 14789949.2010 .506616$.

[11] Widom, C. (1989). The Cycle of Violence. Science, 244(4901), 160-166. Retrieved from http://www. jstor.org/stable/1702789.

[12] Zeanah, Charles H., et al. (2018) Child Abuse and Neglect. Journal of the American Academy of Child \& Adolescent Psychiatry, 57(9), 167-169. 